\title{
Parental Rearing Patterns and Interpersonal Skills in Deaf Chinese College Students: The Mediating Role of Theory of Mind
}

\author{
Yang Wu, Xiping Liu*, Shengnan Zhang and Rubo Zhong \\ Faculty of Psychology, Tianjin Normal University, Tianjin, China
}

This study examined the associations between parental rearing patterns and interpersonal skills via the mediation of Theory of Mind (ToM) in a sample of 369 deaf Chinese college students. The results showed that negative parental rearing patterns were directly and negatively associated with interpersonal skills, and positive parental rearing patterns were directly and positively associated with interpersonal skills. There were also indirectly associated with interpersonal skills via ToM. We also considered whether the mediation

OPEN ACCESS

Edited by:

Weijun Wang,

University at Buffalo, United States

Reviewed by:

Aijuan Cun,

University of New Mexico,

United States

Yuan Yao,

Huaihua University, China

*Correspondence:

Xiping Liu

1xp3771@sina.com

Specialty section:

This article was submitted to Educational Psychology, a section of the journal

Frontiers in Psychology

Received: 13 May 2021 Accepted: 07 July 2021 Published: 20 August 2021

Citation:

Wu Y, Liu X, Zhang S and Zhong $R$ (2021) Parental Rearing Patterns and Interpersonal Skills in Deaf Chinese College Students: The Mediating Role of Theory of Mind.

Front. Psychol. 12:709038.

doi: 10.3389/fpsyg.2021.709038 of ToM was different for male participants and female participants. The indirect associations between parental rearing patterns and interpersonal skills via ToM existed for female participants, but not for male participants. These results indicated that deaf college students' perceived parental rearing patterns are associated with their interpersonal skills, and parents of deaf children should incorporate ToM in their everyday rearing patterns to improve their children's interpersonal skills, especially for girls.

Keywords: parental rearing patterns, Theory of Mind, interpersonal skills, deaf Chinese college students, gender difference

\section{INTRODUCTION}

Interpersonal skills are an important part of intelligence (Brualdi Timmins, 1996) and were defined as "interpersonal competence" by Klein et al. (2006). Interpersonal skills refer to an individual's ability to communicate and interact with different objects through the dynamic verbal (and non-verbal) communication means of complex perceptual and cognitive processes. After entering college, students are exposed to various new social environments, and they are provided more opportunities to interact with their peers and strangers. The social network outside the family gradually expands (Bester, 2019). Developing and maintaining dynamic and stable interpersonal relationships become a key task at this stage. Positive interpersonal skills are often associated with several positive outcomes, including wellbeing, a better quality of life, high academic achievements, higher levels of social adjustment performance, and lower mental health problems (Xia et al., 2015; Madison and Coyne, 2017; Zhang and Eggum-Wilkens, 2018). Conversely, poor interpersonal skills are often associated with negative outcomes, such as loneliness, low self-esteem and confidence, and higher levels of mental health problems (Malaquias et al., 2015; Hamilton et al., 2016; Ranta et al., 2016; Owens et al., 2019). Moreover, this negative correlation is more evident in deaf college students. For example, according to Xiong (2015), 
most interpersonal communication of deaf Chinese students occurs in peer relationships and their interpersonal relationship problems are more severe than those of students with normal hearing. Using a sample of deaf adolescents, Xu (2018) found that deaf adolescents are prone to having conflicts with friends due to the lack of communication skills and their immature concept of friendship, and they are very unlikely to proactively and effectively solve conflicts. With the continuous development of inclusive education, more deaf students live and study with hearing students in colleges. However, due to their deafness, they have difficulties in both general and interpersonal communications. It is very challenging to integrate themselves into society after graduation. Therefore, it is important to study the influencing factors and better understand the mechanisms of interpersonal communication ability in deaf college students.

\section{Parental Rearing Patterns and Interpersonal Skills}

The literature has documented that parental rearing patterns are an important factor affecting interpersonal skills of deaf individuals (Darling and Steinberg, 1993; Huang, 2010; Xiuqin et al., 2010; Llorca et al., 2017). Darling and Steinberg (1993) believe that parental rearing patterns shape the attitude that parents pass on to their children. The emotional climate created by parents' behavior includes not only behavioral goals but also non-goal-oriented behaviors, such as changes in normal posture, intonation, gesture, and emotions. Parents are children's first teachers, and their parental rearing patterns will affect children's personality formation and social interaction (Huang, 2010). For example, adolescents with Internet addiction disorder generally perceive maternal and paternal rearing styles as lacking in emotional warmth, being over-involved, displaying rejection, and being punitive (mothers only). Internet-addicted adolescents also rate higher on obsessiveness, interpersonal sensitivity, depression, and paranoid ideation (Xiuqin et al., 2010). The mother's permissive style is associated with the increased aggressive behavior and decreased attachment to peers (Llorca et al., 2017).

Parents of deaf children are more likely to adopt inappropriate parental rearing patterns, such as excessive compensation or additional refusal, as a result of their children's physical disability. They often fail to provide their deaf children appropriate education guidance. Some tend to be more indulgent regarding their children's education; some are overprotective and participate excessively in their children's daily lives, negatively impacting the psychological growth of deaf adolescents (Kang, 2018). Gao (2014) found that parents of deaf children pay too much attention to their children's personal needs and self-care ability instead of their connection with the outside world, thereby hindering the normal social development of deaf children. At the same time, a one-way and forced communication practice makes deaf children most likely to adopt a passive position in interpersonal communication, which is not conducive to their future interpersonal relationships. However, although research has indicated associations between parental rearing patterns and interpersonal skills in deaf adolescents, few studies have examined the relationship in deaf college students.

\section{The Mediating Role of Theory of Mind}

Theory of Mind (ToM) is a term for attributing a psychological state to oneself or others and predicting and explaining human behavior (Premack and Woodruff, 1978), the ability to understand the inner world of others. Social constructivism holds that children construct their understanding of others by participating in social interaction mediated by language (Fernyhough, 2008). This social interaction includes the interaction between parents and children. It is possible that ToM mediates the relationship between parental rearing patterns and interpersonal skills. First, some studies have shown that ToM is a key factor in the development of interpersonal skills and in the formation and maintenance of social relations (Devine and Hughes, 2016; Lecciso et al., 2016; Petrocchi et al., 2021). For example, in kindergarteners, higher ToM was related to stronger social skills (Razza and Blair, 2009). Poor ToM may lead to being a victim or a bully in early adolescence (Happé et al., 2013). Second, some studies have shown that proper parenting styles include emotional involvement and equal dialogue with children; however, inappropriate parenting styles (such as high involvement or rejection) make it difficult to have such a dialogue between parents and children (Fuentes et al., 2015; Lorence et al., 2020). Verbal dialogue is an essential part of parenting (Keller et al., 2008).

Studies have also shown that dialogue between family members is helpful for adolescents to think in transposition (Weber and Carter, 1998). Children who disclose personal information to family members receive high scores in the ToM task (Rotenberg, 2010). When family conversations involve states of mind, there will be discussion on the differences in the thoughts of oneself and others. Children are aware of thoughts, memories, and beliefs, and all of these affect the development of ToM (Dunn et al., 1991). However, this kind of family dialogue raises questions as to whether there is a common language in the family and in the rearing patterns of parents of deaf children. First, deaf children who grow up in a hearing environment may not have a common language at home; they may not be able to use sign language to communicate. This would result in limited opportunities for deaf children to participate in various dialogues, which, for example, include different views and use of mental state terms (such as know, believe, and think; Moeller and Schick, 2006), and a series of syntactic structures (De Villiers and De Villiers, 2014). Although many deaf children's parents also learn sign language, they may not be fluent in it. Thus, the early language environment of deaf children may not be as good as that of hearing children (Moeller and Schick, 2006). Compared with deaf children growing up in deaf families, deaf children growing up in hearing families have less conversation at home (e.g., Peterson and Slaughter, 2006). Parents' conversation with deaf children lacks high-level facilitating discourse (such as the use of psychological terms) and is more indicative than their conversation with hearing children (Ambrose et al., 2015). This situation often results in deaf children being unable to talk freely to their parents about their observations, feelings, and other psychological states. Their ToM can be as retarded as that of autistic children. Second, parents may not have much patience to build a warm upbringing environment in which their deaf children could 
discuss their psychological state, and that of others. It may be difficult for parents who often refuse and deny their children and are indifferent to their children's feelings to create a family environment where they could have a psychological dialogue. This study aimed to examine whether ToM would mediate the relationship between parental rearing patterns and interpersonal skills among deaf Chinese college students. Such a model would make a good contribution to the literature.

\section{Gender Difference}

Studies have indicated gender differences in ToM at children's different stages of development. Preschool girls have shown a slight advantage over boys in emotional understanding and false belief tasks (Banerjee, 1997; Charman et al., 2002; Walker, 2005). Xu and Ge (2010) used low-level and high-level ToM stories to test whether there were gender differences in college students' ToM levels. They found that there were significant gender differences in low-level tasks (but not in high-level tasks); that is, girls' understanding of other people's beliefs and white lie stories was better than that of boys. Wu (2014) tested ToM levels of adults at different age stages and found that females' scores of ToM were higher than those of males in all three age groups (youth, middle age, and old age) and that there was gender difference in young people's ToM (but not in the other two age groups). Additionally, this gender difference was confirmed in neuroimaging. Researchers (e.g., Derntl et al., 2010; Gao et al., 2019) divided ToM into cognitive dimension (cognitive ToM/cToM) and affective dimension (affective ToM/aToM) and tested gender differences in brain nerve responses in these two dimensions in adolescents. According to Gao et al. (2019), when completing the cognitive ToM task, the activity of left temporoparietal junction in male adolescents was significantly higher than that of female adolescents. Derntl et al. (2010) found that when inferring emotional responses from graphic scenes, men were more likely than women to activate the temporoparietal junction. However, when inferring emotions, women were more likely than men to activate the amygdala, inferior frontal gyrus, and superior temporal sulcus. This study considered whether there would be gender differences, if our proposed mediation model via ToM existed.

\section{The Present Study}

The present study was designed to examine the associations between parental rearing patterns and interpersonal skills within a sample of Chinese deaf college students. We hypothesized that negative parental rearing patterns will be negatively associated with interpersonal skills, whereas positive parental rearing patterns will be positively associated with interpersonal skills. We also considered whether the relationship between parental rearing patterns and interpersonal skills would be mediated via ToM. We expected that deaf college students who reported lower perceived negative parental rearing patterns would report higher ToM, which in turn would be positively associated with interpersonal skills. Higher positive parental rearing patterns were expected to be associated with increased interpersonal skills due to its association with ToM. We also considered whether the relationship between parental rearing patterns and interpersonal skills via ToM was different for male participants and female participants but offered no hypothesis.

\section{MATERIALS AND METHODS}

\section{Participants and Recruitment}

The sample included 369 deaf college students $(M=19.94$ years old, $S D=1.52 ; 50.1 \%$ female) at a special education college in the northern China. During the Spring 2020 semester, 380 hearing-impaired students were invited to participate in a research study of student behaviors and beliefs. To be eligible, students had to be 17 years or older with hearing loss. They had to have passed the college entrance test and have a proficient level of written and spoken Chinese or Chinese sign language. All students had passed the admission mental health examination and had not been clinically diagnosed with any mental disorder. Eleven students did not attend the study.

\section{Procedure and Measures}

Student assent and written informed consent from parents were obtained. Participation in the study was voluntary, and no economic remuneration was provided. After providing the informed consent, students proceeded to fill the paper-and-pencil survey questionnaire during the regular class hours. Participants were randomly assigned to either classrooms. Questionnaires were administered in each of these classrooms by two trained researchers and one interpreter proficient in Chinese sign language. We believed that this process would reduce the pressure of sign language translation and improve the testing quality. When the deaf college participants had difficulties with the written language, they could seek help from the sign language interpreter. The survey questionnaire was completed in $60 \mathrm{~min}$. All procedures were approved by the University Research Ethics Board (Institutional Review Board).

Parental rearing patterns were measured using the adapted version of the Short-Egna Minnen av Barndoms Uppfostran (S-EMBU; Arrindell et al., 1999; Jiang et al., 2010). The S-EMBU includes 21 items and assesses students' perceptions of their mother's and father's behavior toward them. These included six items on rejection (e.g., "My father/mother punishes me for even the smallest mistakes."), eight items on overprotection (e.g., "I feel my parents interfere in everything I do."), and seven items on emotional warmth (e.g., "Father/mother praises me."), rated on 4-point scales, from never (1) to always (4). We took the average of the rejection and overprotection items to compute a negative parental rearing patterns variable ( $\alpha=0.83$ ), and the emotional warmth items were averaged to create a positive parental rearing patterns variable $(\alpha=0.86)$ for each participant.

Interpersonal skills were measured using the 40-item Interpersonal Competence Questionnaire developed by psychologists from the University of California (Wei, 2005). The questionnaire assesses college students' proactive communication (eight items; e.g., "Start a conversation with 
a stranger you'd like to meet."), appropriate refusal (eight items; e.g., "Reject unreasonable demands from people close to you."), self-disclosure (eight items; e.g., "Tell your loved ones something about yourself that you are ashamed of."), conflict management (eight items; e.g., "Able to open up feelings of jealousy or resentment during an argument with someone close to you."), and emotional support (eight items; e.g., "To help close people think and experience their major life decisions."), rated on a 5-point scale, from 1 (can't do, feel extremely uneasy and not sure, and try to escape) to 5 (very good at doing this, feel very relaxed, and can handle very well). We took the sum of all these 40 items to create an interpersonal skills variable for each participant $(\alpha=0.92)$.

Theory of Mind was measured using the adapted version of the advanced Theory-of-Mind tasks (AToM; Osterhaus et al., 2016). The AToM assesses participants' social reasoning (nine items; e.g., "Why does Mrs. Smith say that?"), fuzzy reasoning (three items; e.g., "What did Maomao see in the picture?"), and the understanding of deviant behavior from social norms (three items; e.g., "Did Johnny remember the birthday party was a surprise?"). The scale consists of a series of ToM stories and pictures, and following each of these, one or more questions were provided. There were 15 questions in total, and the responses were Yes (1) or No (0). We summed all these 15 responses to produce a ToM score for each participant $(\alpha=0.70)$.

\section{Analytic Plan}

We hypothesized that negative parental rearing patterns would be negatively associated with deaf college students' interpersonal skills, whereas positive parental rearing patterns would be positively associated with their interpersonal skills. We also hypothesized that the relationship between parental rearing patterns and interpersonal skills would be mediated via ToM. We tested the direct and indirect effects of parental rearing patterns on interpersonal skills in Mplus Version 8.6 (Muthén et al., 2016; Muthén and Muthén, 2017). We used maximum likelihood estimation with robust standard errors. The statistical significance of the indirect effect was tested using 50,000 bootstrap draws to estimate precisely the $95 \%$ confidence intervals determined from the lower and upper 2.5 percentiles (MacKinnon et al., 2004; Hayes, 2013). Participants' gender ( 0 = male; 1 = female $)$ and age were included as covariates, allowing us to control for their influence on the outcome (i.e., interpersonal skills) and on the mediator (i.e., ToM) in the model (see Figure 1). We also considered whether the paths from parental rearing patterns to interpersonal skills via ToM were different for male participants and female participants. Thus, we conducted multiple group analysis (Muthén and Muthén, 2017).

\section{RESULTS}

\section{Descriptive Data}

The descriptives and bivariate correlations among primary variables are displayed in Table 1. Independent samples t-test did not indicate gender differences in the primary variables, that is, interpersonal skills, perceived parental rearing patterns, ToM, or age.

\section{Are the Effects of Parental Rearing Patterns on Interpersonal Skills Mediated via ToM?}

We tested the direct and indirect effects of perceived negative parental rearing patterns and positive parental rearing patterns on interpersonal skills. As shown in Figure 1, negative parental rearing patterns were negatively associated with ToM, which in turn was associated with higher interpersonal skills. The indirect effect of negative parental rearing patterns via ToM was significant, $b=-0.002$ (0.001), $p=0.007$, and 95\% bootstrap confidence intervals did not include the value of $0(-0.003,0.000), b=-0.002(0.001), p=0.009$. Negative parental rearing patterns were also directly and negatively associated with interpersonal skills, $b=-0.008$ (0.003), $p=0.002$. The total estimated effect from negative parental rearing patterns to interpersonal skills was $b=-0.010$ (0.002), $p<0.001$. Positive parental rearing patterns were positively associated with ToM, which in turn was associated with higher interpersonal skills. The path via ToM was significant, $b=0.002$ (0.001), $p=0.011$, and 95\% bootstrap confidence intervals did not include the value of $0(0.000,0.004)$, $b=0.002$ (0.001), $p=0.013$. Positive parental rearing patterns were also directly and positively associated with interpersonal skills, $b=0.020$ (0.004), $p<0.001$. The total estimated effect from positive parental rearing patterns to interpersonal skills was $b=0.022$ (0.004), $p<0.001$. The model controlled for gender and age on the outcome (i.e., interpersonal skills) and the mediator (i.e., ToM). The effect of gender was not significant on ToM $(p=0.684)$ or on interpersonal skills $(p=0.696)$. Age had a significant effect on ToM, $b=0.508$ (0.129), $p<0.001$, but not on interpersonal skills $(p=0.509)$. The model fit indices were good: $\chi^{2}(4)=5.721, p=0.221$; $\mathrm{CFI} / \mathrm{TLI}=0.986 / 0.968 ; \mathrm{RMSEA}=0.034 ; \mathrm{SRMR}=0.031$.

\section{Gender Difference in the Mediation Role of ToM}

We conducted multiple group analysis to examine whether the paths via ToM were different for male participants and female participants. Results showed good model fit: $\chi^{2}(4)=5.121, p=0.275 ;$ CFI $/$ TLI $=0.993 / 0.975$; RMSEA $=$ 0.039; SRMR $=0.035$. As shown in Figure 2, for female participants, ToM mediated the relationship between negative parental rearing patterns and interpersonal skills, $b=-0.004$ (0.001), $p=0.008$ (95\% bootstrap confidence intervals were $-0.006,-0.001)$ and also the relationship positive parental rearing patterns and interpersonal skills, $b=0.006(0.002)$, $p=0.001$ (95\% bootstrap confidence intervals were 0.002 , $0.010)$. ToM did not mediate the path from negative parental rearing patterns to interpersonal skills or from positive parental rearing patterns to interpersonal skills for male participants. 


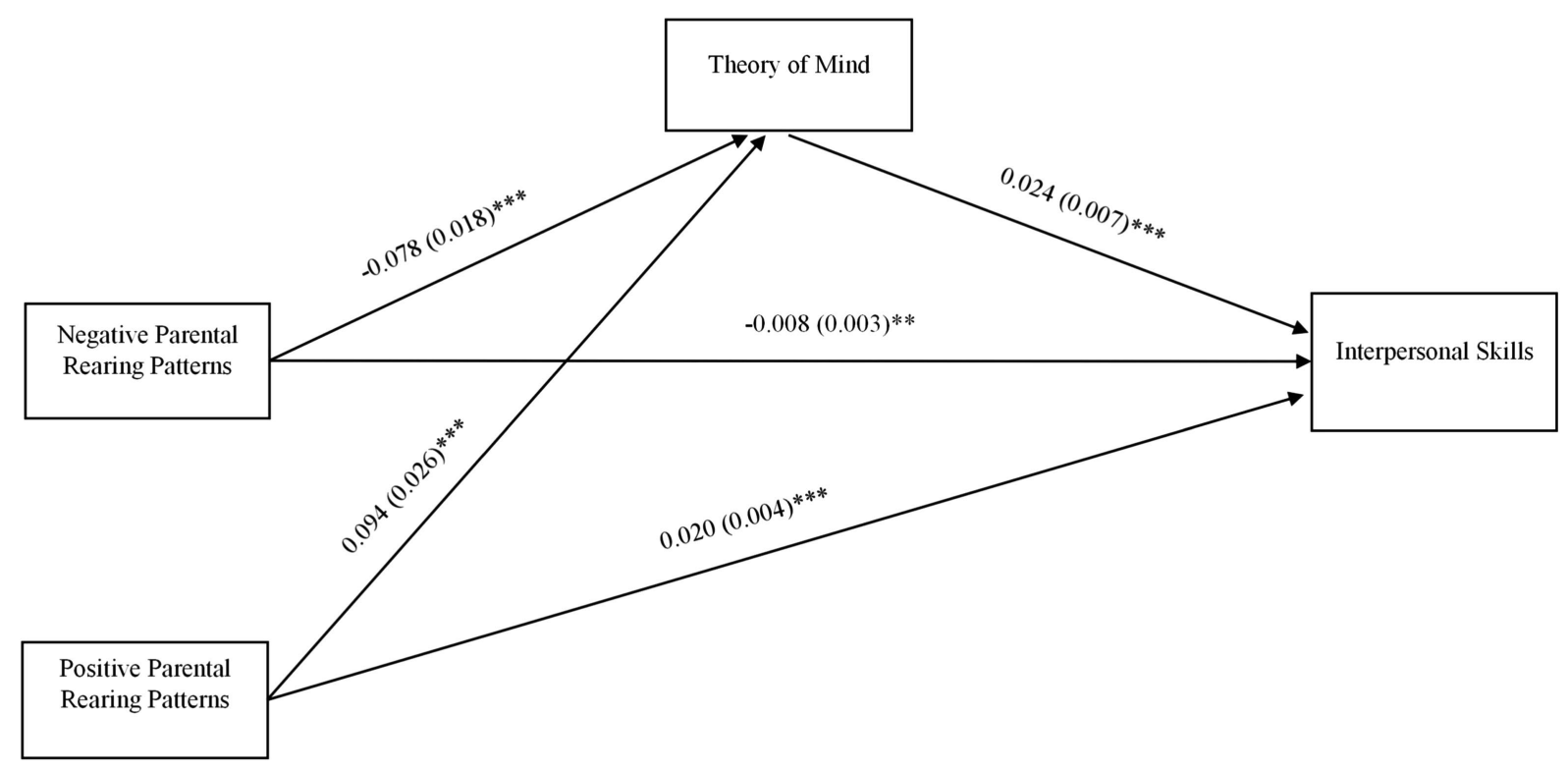

FIGURE 1 | Indirect effects of parental rearing patterns on interpersonal skills via Theory of Mind (ToM). Path coefficients (b and standard error, SE) are unstandardized. Model controlled for potential effects of gender and age on ToM and interpersonal skills. ${ }^{* * * *} p<0.001 ;{ }^{* *} p<0.01 ; "{ }^{*} p<0.05$.

\section{DISCUSSION}

This study examined the relationship between parental rearing patterns and interpersonal skills and its mechanism via ToM in a sample of deaf Chinese college students. Firstly, parenting styles were significantly associated with ToM, which in turn was associated with higher interpersonal skills; Secondly, ToM played a mediating role in the relationship between parental rearing patterns and interpersonal communication ability. However, multiple grouping modeling indicated that this mediation role of ToM was observed only for female participants (but not for male participants).

\section{Relationship Between Parental Rearing Patterns and Interpersonal Communication Ability in Deaf College Students}

This study found that parental rearing patterns were significantly associated with interpersonal ability of deaf Chinese college students. Positive parental rearing patterns were positively associated with interpersonal skills, while negative parental rearing patterns were negatively associated with interpersonal skills. The results are consistent with previous studies (Liu et al., 2018). Social learning theory holds that children interact directly with parents by observing their parents' interaction patterns or by learning from parents under their guidance (Crittenden, 1984). The learning includes attitudes, values, personality, behavior, and social emotions (Dohmen et al., 2012; Schofield et al., 2012; Barni et al., 2013). Parents' rearing patterns are an interactive way for children to learn from their parents. Through this interaction, parents convey their interpersonal relationship model to their children. Thus, interpersonal and intergenerational transmission occurs.
Some studies have shown that the parent-child relationship and especially the fathers' rearing pattern explain the advantages of children's interpersonal relationships (Conger et al., 2010). Parents with emotional warmth will show an interest in their children's activities and opinions. They will talk about their feelings and emotions with them, provide comfort, moral guidance, and advice, participate in social activities with them, listen to them carefully, and help them solve problems. This warm upbringing approach is related to positive mental health, social ability, self-esteem, academic achievement, and healthy peer relationships (BoudreaultBouchard et al., 2013; Grolnick et al., 2015). The more childhood memories of parental rejection individuals have, the more likely they are to experience psychological disorders (Rohner and Khaleque, 2010), and these negative memories will compromise the development of their interpersonal ability. Such conclusions have also been demonstrated in cross-cultural studies (Ali et al., 2015).

\section{Mediating Role of ToM and the Gender Difference}

This study found that ToM plays a mediating role in the relationship between parental rearing patterns and interpersonal communication ability in deaf college students. This suggests that parents' positive parental rearing patterns are conducive to improving the level of ToM of deaf college students, which will make it easier for them to transpose thinking and to understand individual psychological differences by observing other people's external behavior. This will help them to improve their interpersonal skills and to conduct interpersonal communication calmly and appropriately. However, deaf college students' perceived negative parental rearing patterns will lead to the lagging development of their ToM, making it difficult to integrate themselves into normal interpersonal relationships.

In families with deaf children, due to the physical disability of the children, parents may be prone to overintervention and 


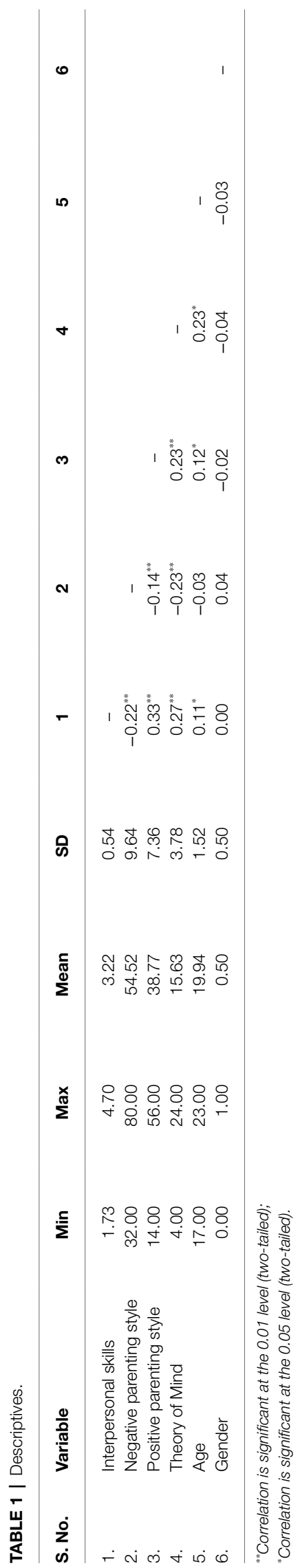

overprotection in their parenting because they may experience guilt about their children. In addition, deaf children growing up in hearing families may have difficulty in effective communication to promote psychological growth due to the lack of a common language with their parents. Studies have shown that communication with parents is an important predictor of group identification and socialization standard for deaf persons (Calderon, 2000). The development of ToM is influenced by a number of external environmental factors, among which the family environment is particularly important. Devine and Hughes (2016) concluded in a meta-analysis that parents' psychological conversation and their way of thinking are significantly correlated with their children's ToM level. Even after controlling for the language factors, these correlations still exist. Social constructivist theorists believe that increasing the discussion of mental states provides children with more opportunities to understand other people's thoughts, thereby testing and revising their ToM (Jenkins et al., 2003; Wellman, 2017). The improvement and development of ToM skills can support the ability to sympathize with others (Peterson, 2016), help individuals to have more friends (Peterson and Siegal, 2002; Fink et al., 2015), and even affect peer popularity (Peterson, 2016).

This study also found that ToM mediated the relationship between negative/positive parental rearing patterns and interpersonal skills only for female deaf college students. There are two possible reasons for this disparity. First, girls' understanding of psychological theory has been ahead of boys since childhood (Walker, 2005; Wu, 2014). Behavioral studies show that women perform better than men in social sensitivity, empathy, and emotional intelligence tasks (McClure, 2000; Baron-Cohen and Wheelwright, 2004; Brackett and Salovey, 2006). These abilities are closely related to the level of ToM. Gender difference in ToM is also reflected in brain activity. Neuroimaging studies have shown that compared with men of the same age, women use additional brain regions in the ToM task for emotion and self-reference thinking (ChristovMoore et al., 2014). Frank et al. (2015) found that in the process of false belief reasoning, women are more likely than men to activate the medial prefrontal cortex. Second, Chinese social culture requires men to appear stronger and more independent. Therefore, parents are more inclined to adopt a free-range parental rearing patterns for boys. As a result, parental rearing patterns may have no significant impact on boys' interpersonal skills. When girls are young, they have more advantages in emotion recognition and expression than boys. Gender differences in the level of ToM may further promote the interaction between parents and girls, and girls may gain more attention from parents, resulting in significant gender differences in the mediating role of ToM between parental rearing patterns and interpersonal skills.

\section{Limitations and Prospects}

There are some limitations in this study. First, the relationship model in this study was based on a cross-sectional design. Future studies should collect multiple points of data and combine both vertical and horizontal research results to replicate and better understand the proposed relationship model which we examined in a sample of deaf Chinese college students. Second, this study showed gender differences in the mediating effect of ToM; that is, ToM mediated the relationship between parental rearing 


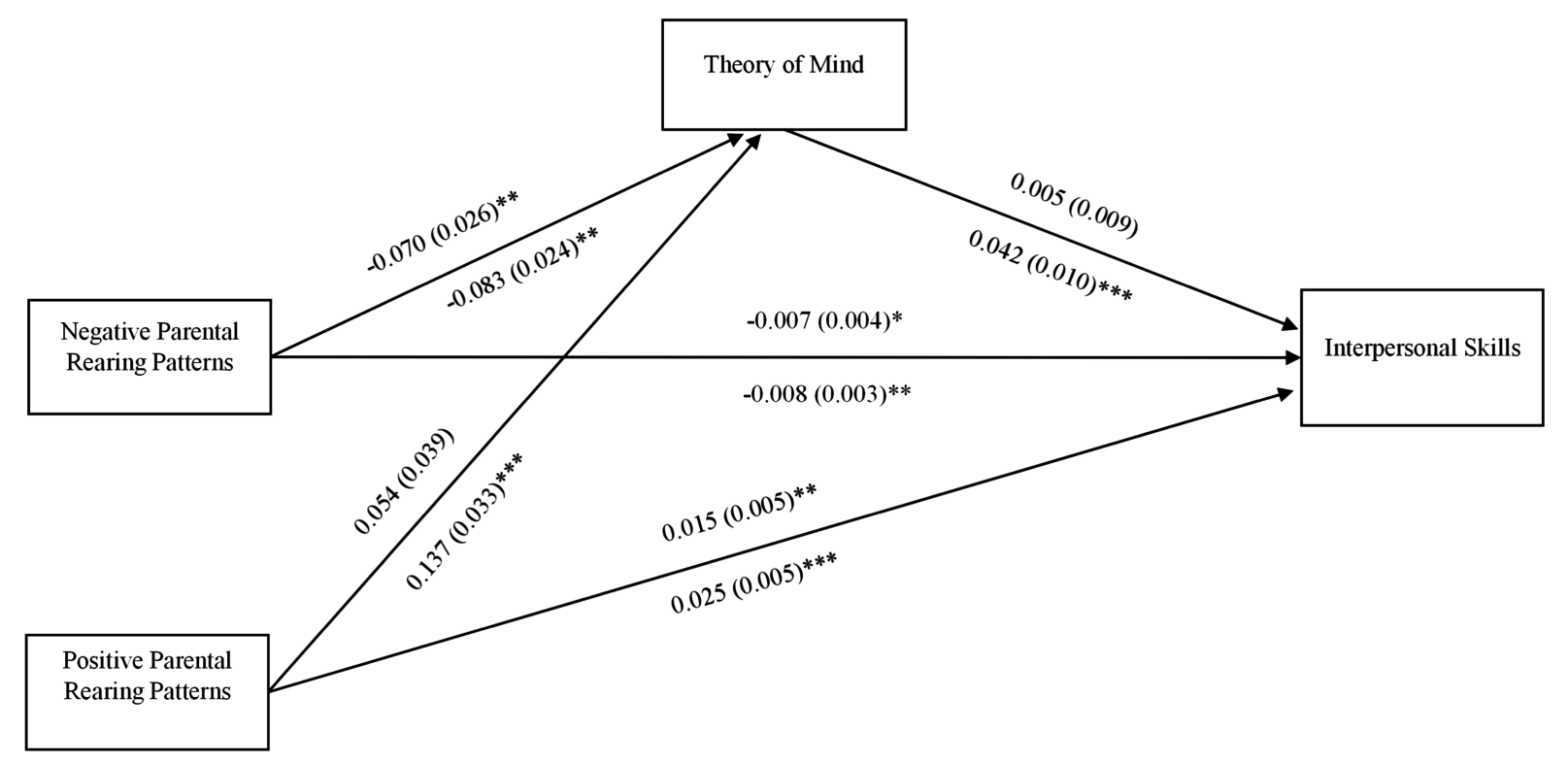

FIGURE 2 | Indirect effects of parental rearing patterns on interpersonal skills via ToM, by gender. Path coefficients (b and standard error, SE) are unstandardized. Path coefficients above the arrows were for male participants, and those below the arrows were for female participants. Model controlled for potential effect of age on ToM and interpersonal skills. ${ }^{* * *} p<0.001 ; " * 0<0.01 ; " p<0.05$.

patters and interpersonal skills only for female participants. However, future research should replicate it and we also encourage these findings should be replicated in adolescents. Third, the data in this study were participants self-reported. Future research could use a variety of data collection methods to obtain more accurate results, for example, including the perceptions of parents themselves. Finally, the data were collected from deaf students at only one college. The findings should be interpreted with caution.

\section{Educational Enlightenment}

First, schools should increase their interactions with the parents of the students with hearing loss and better understand these students' parental rearing patterns and their influences on their children's everyday practice. Parents should be respectfully educated about inappropriate parental rearing patterns using appropriate, evidence-based programs. Second, deaf college students should be trained with a special attention paid to the improvement of their ToM, which could be implemented in a targeted and phased way through daily management and group counseling. For example, for those deaf students whose ToM' level is lagging, targeted training should be available to help them to integrate better into society. Third, parents of deaf children should be educated to avoid adopting overprotective parental rearing patterns with scientific research evidence. In rearing deaf children, parents should be encouraged to learn sign language as much as possible, and their children should wear a hearing-aid/cochlear implantation (CI) as early as possible, thereby reducing the communication barriers between parents and children. Furthermore, parents should be encouraged to pay attention to the development of children's ToM and help children to improve the level of ToM through effective communication. Social support should be available for these parents and families. Finally, although this study did not observe a significant mediating role of ToM for male students, and for female and male deaf college students, different management and guidance methods may be adopted according to their individual characteristics, we believe that warm teacher-student relationships and peer relationships can help students to integrate into groups and can improve their interpersonal skills.

\section{DATA AVAILABILITY STATEMENT}

The raw data supporting the conclusions of this article will be made available by the authors, without undue reservation.

\section{ETHICS STATEMENT}

All procedures were approved by the University Research Ethics Board (Institutional Review Board). Student assent and written informed consent from parents were obtained. Written informed consent was obtained from the individuals for the publication of any potentially identifiable images or data included in this article.

\section{AUTHOR CONTRIBUTIONS}

YW analyzed the data and wrote the materials and methods, results, and discussion sections. XL and SZ contributed to the conception and design of the study. $\mathrm{RZ}$ is responsible for the drafting the work and revising it critically for important intellectual content. All authors contributed to the article and approved the submitted version. 


\section{REFERENCES}

Ali, S., Khaleque, A., and Rohner, R. P. (2015). Influence of perceived teacher acceptance and parental acceptance on youth's psychological adjustment and school conduct: A cross-cultural meta-analysis. Cross-Cult. Res. 49, 204-224. doi: $10.1177 / 1069397114552769$

Ambrose, S. E., Walker, E. A., Unflat-Berry, L. M., Oleson, J. J., and Moeller, M. P. (2015). Quantity and quality of caregivers' linguistic input to 18-month and 3-year-old children who are hard of hearing. Ear Hear. 36, 48-59. doi: 10.1097/AUD.0000000000000209

Arrindell, W. A., Sanavio, E., Aguilar, G., Sica, C., Hatzichristou, C., Eisemann, M., et al. (1999). The development of a short form of the EMBU 1: its appraisal with students in Greece, Guatemala, Hungary and Italy. Pers. Individ. Differ. 27, 613-628. doi: 10.1016/S0191-8869(98)00192-5

Banerjee, M. (1997). Hidden emotions: Preschoolers' knowledge of appearancereality and emotion display rules. Soc. Cogn. 15, 107-132. doi: 10.1521/ soco.1997.15.2.107

Barni, D., Alfieri, S., Marta, E., and Rosnati, R. (2013). Overall and unique similarities between parents' values and adolescent or emerging adult children's values. J. Adolesc. 36, 1135-1141. doi: 10.1016/j.adolescence.2013.09.002

Baron-Cohen, S., and Wheelwright, S. (2004). The empathy quotient: an investigation of adults with Asperger syndrome or high functioning autism, and normal sex differences. J. Autism Dev. Disord. 34, 163-175. doi: 10.1023/B: JADD.0000022607.19833.00

Bester, G. (2019). Stress experienced by adolescents in school: the importance of personality and interpersonal relationships. J. Child Adolesc. Ment. 31, 25-37. doi: 10.2989/17280583.2019.1580586

Boudreault-Bouchard, A. M., Dion, J., Hains, J., Vandermeerschen, J., Laberge, L., and Perron, M. (2013). Impact of parental emotional support and coercive control on adolescents' self-esteem and psychological distress: results of a four-year longitudinal study. J. Adolesc. 36, 695-704. doi: 10.1016/j. adolescence.2013.05.002

Brackett, M. A., and Salovey, P. (2006). Measuring emotional intelligence using the Mayers-Salovey-Caruso emotional intelligence test. Psicothema 18, 34-41.

Brualdi Timmins, A. C. (1996). Multiple intelligences: Gardner's theory. PARE 5:10.

Calderon, R. (2000). Parental involvement in deaf children's education programs as a predictor of child's language, early reading, and social-emotional development. J. Deaf. Stud. Deaf. Educ. 5, 140-155. doi: 10.1093/deafed/5.2.140

Charman, T., Ruffman, T., and Clements, W. (2002). Is there a gender difference in false belief development? Soc. Dev. 11, 1-10. doi: 10.1111/1467-9507.00183

Christov-Moore, L., Simpson, E. A., Grigaityte, K., Iacoboni, M., Ferrari, P. F., Sciences, B., et al. (2014). Empathy: Gender effects in brain and behaviour. Neurosci. Biobehav. Rev. 46, 604-627. doi: 10.1016/j.neubiorev.2014.09.001

Conger, R. D., Conger, K. J., and Martin, M. J. (2010). Socioeconomic status, family processes, and individual development. J. Marriage Fam. 72, 685-704. doi: $10.1111 /$ j.1741-3737.2010.00725.x

Crittenden, P. M. (1984). Sibling interaction: Evidence of a generational effect in maltreating infants. Child Abuse Negl. 8, 433-438. doi: 10.1016/ 0145-2134(84)90024-3

Darling, N., and Steinberg, L. (1993). Parenting style as context: An integrative model. Psychol. Bull. 113, 487-496. doi: 10.1037/0033-2909.113.3.487

De Villiers, J. G., and De Villiers, P. A. (2014). The role of language in theory of mind development. Top. Lang. Disord. 34, 313-328. doi: 10.1097/ TLD.0000000000000037

Derntl, B., Finkelmeyer, A., Eickhoff, S., Kellermann, T., Falkenberg, D. I., Schneider, F., et al. (2010). Multidimensional assessment of empathic abilities: neural correlates and gender differences. Psychoneuroendocrinology 35, 67-82. doi: $10.1016 /$ j.psyneuen.2009.10.006

Devine, R. T., and Hughes, C. (2016). Measuring theory of mind across middle childhood: Reliability and validity of the silent films and strange stories tasks. J. Exp. Child Psychol. 149, 23-40. doi: 10.1016/j.jecp.2015.07.011

Dohmen, T., Falk, A., Huffman, D., and Sunde, U. (2012). The intergenerational transmission of risk and trust attitudes. Rev. Econ. Stud. 79, 645-677. doi: $10.1093 /$ restud/rdr027

Dunn, J., Brown, J., and Beardsall, L. (1991). Family talk about feeling states and children's later understanding of other' emotions. Dev. Psychol. 27, 448-455. doi: 10.1037/0012-1649.27.3.448
Fernyhough, C. (2008). Getting Vygotskian about theory of mind: mediation, dialogue, and the development of social understanding. Dev. Rev. 28, 225-262. doi: 10.1016/j.dr.2007.03.001

Fink, E., Begeer, S., Peterson, C. C., Slaughter, V., and de Rosnay, M. (2015). Friendlessness and theory of mind: A prospective longitudinal study. $\mathrm{Br}$. J. Dev. Psychol. 33, 1-17. doi: 10.1111/bjdp.12060

Frank, C. K., Baron-Cohen, S., and Ganzel, B. L. (2015). Sex differences in the neural basis of false-belief and pragmatic language comprehension. NeuroImage 105, 300-311. doi: 10.1016/j.neuroimage.2014.09.041

Fuentes, M. C., Alarcón, A., Gracia, E., and Garcia, F. (2015). El ajuste escolar en los adolescentes españoles: Influencia de la socialización parental [School adjustment among Spanish adolescents: Influence of parental socialization]. Cult. Educ. 27, 1-32. doi: 10.1080/11356405.2015.1006847

Gao, H. (2014). Investigation and study on the status of parent-child communication between families of hearing-impaired children in Hohhot. J. Imnu. 27, 65-67. doi: 10.3969/j.issn.1671-0916.2014.12.024

Gao, Y., Rogers, J. C., Pauli, R., Clanton, R., Baker, R., Birch, P., et al. (2019). Neural correlates of theory of mind in typically-developing youth: influence of sex, age and callous-unemotional traits. Sci. Rep. 9, 16216-16212. doi: 10.1038/s41598-019-52261-y

Grolnick, W. S., Raftery-Helmer, J. N., Flamm, E. S., Marbell, K. N., and Cardemil, E. V. (2015). Parental provision of academic structure and the transition to middle school. J. Res. Adolesc. 25, 668-684. doi: 10.1111/ jora.12161

Hamilton, J. L., Potter, C. M., Olino, T. M., Abramson, L. Y., Heimberg, R. G., and Alloy, L. B. (2016). The temporal sequence of social anxiety and depressive symptoms following interpersonal stressors during adolescence. J. Abnorm. Child Psychol. 44, 495-509. doi: 10.1007/s10802-015-0049-0

Happé, F., Moffitt, T. E., and Arseneault, L. (2013). A prospective longitudinal study of children's theory of mind and adolescent involvement in bullying J. Child Psychol. Psychiatry 53, 254-261. doi: 10.1111/j.1469-7610.2011.02488.x.A

Hayes, A. F. (2013). Introduction to Mediation, Moderation, and Conditional Process Analysis: A Regression-Based Approach. New York: The Guilford Press.

Huang, J. (2010). Parental parenting has an impact on children's interpersonal relationships. Sci. Inf. 20, 89-93. doi: 10.3969/j.issn.1001-9960.2010.20.396

Jenkins, J. M., Turrell, S. L., Kogushi, Y., Lollis, S., and Ross, H. S. (2003). A longitudinal investigation of the dynamics of mental state talk in families. Child Dev. 74, 905-920. doi: 10.1111/1467-8624.00575

Jiang, J., Lu, Z., Jiang, B., and Xu, Y. (2010). Preliminary revision of the Chinese version of the simplified parenting style questionnaire. Psychol. Dev. Educ. 26, 94-99.

Kang, K. (2018). Social participation of hearing-impaired youth in the new media environment: problems and professional reflection. Soc. Work. Chn. 25, 26-27. doi: 10.3969/j.issn.1674-3857.2018.25.013

Keller, H., Otto, H., Lamm, B., Yovsi, R. D., and Kärtner, J. (2008). The timing of verbal/vocal communications between mothers and their infants: A longitudinal cross-cultural comparison. Infant Behav. Dev. 31, 217-226. doi: 10.1016/j.infbeh.2007.10.001

Klein, C., DeRouin, R. E., and Salas, E. (2006). “Uncovering workplace interpersonal skills: a review, framework, and research agenda," in International Review of Industrial and Organizational Psychology. eds. G. P. Hodgkinson and J. K. Ford (West Sussex, England: John Wiley \& Sons, Ltd.), 79-126.

Lecciso, F., Levante, A., Baruffaldi, F., and Petrocchi, S. (2016). Theory of mind in deaf adults. Cogent Psychol. 3, 1-14. doi: 10.1080/23311908.2016.1264127

Liu, L., Xu, L., Luo, F., and Li, Y. (2018). Intergenerational transmission of interpersonal strengths: The role of parent gender, family processes, and child characteristics. J. Adolesc. 67, 66-76. doi: 10.1016/j.adolescence.2018.06.005

Llorca, A., Cristina Richaud, M., and Malonda, E. (2017). Parenting, peer relationships, academic self-efficacy, and academic achievement: direct and mediating effects. Front. Psychol. 8:2120. doi: 10.3389/fpsyg.2017.02120

Lorence, B., Nunes, C., Menéndez, S., Pérez-Padilla, J., and Hidalgo, V. (2020). Adolescent perception of maternal practices in Portugal and Spain: similarities and differences. Sustainability 12, 1-14. doi: 10.3390/su12155910

MacKinnon, D. P., Lockwood, C. M., and Williams, J. (2004). Confidence limits for the indirect effect: distribution of the product and resampling methods. Multivar. Behav. Res. 39, 99-128. doi: 10.1207/s15327906mbr3901_4 
Madison, L. M. P., and Coyne, K. M. S. M. (2017). Associations between prosocial and problem behavior from early to late adolescence. J. Youth Adolesc. 47, 961-975. doi: 10.1007/s10964-017-0736-y

Malaquias, S., Crespo, C., and Francisco, R. (2015). How do adolescents benefit from family rituals? Links to social connectedness, depression and anxiety. J. Child Fam. Stud. 24, 3009-3017. doi: 10.1007/s10826-014-0104-4

McClure, E. B. (2000). A meta-analytic review of sex differences in facial expression processing and their development in infants, children, and adolescents. Psychol. Bull. 126:424. doi: 10.1037/0033-2909.126.3.424

Moeller, M. P., and Schick, B. (2006). Relations between maternal input and theory of mind understanding in deaf children. Child Dev. 77, 751-766. doi: 10.1111/j.1467-8624.2006.00901.x

Muthén, L. K., and Muthén, L. K. (2017). Mplus Users' Guide. 8th Edn. Los Angeles, CA: Muthén \& Muthén.

Muthén, B. O., Muthén, L. K., and Asparouhov, T. (2016). Regression and Mediation Analysis Using Mplus. Los Angeles, CA: Muthén \& Muthén.

Osterhaus, C., Koerber, S., and Sodian, B. (2016). Scaling of advanced theoryof-mind tasks. Child Dev. 87, 1971-1991. doi: 10.1111/cdev.12566

Owens, S. A., Helms, S. W., Rudolph, K. D., Hastings, P. D., Nock, M. K., and Prinstein, M. J. (2019). Interpersonal stress severity longitudinally predicts adolescent girls' depressive symptoms: the moderating role of subjective and HPA axis stress responses. J. Abnorm. Child Psychol. 47, 895-905. doi: 10.1007/s10802-018-0483-x

Peterson, C. C. (2016). Empathy and theory of mind in deaf and hearing children. J. Deaf. Stud. Deaf. Educ. 21, 141-147. doi: 10.1093/deafed/ env058

Peterson, C. C., and Siegal, M. (2002). Mindreading and moral awareness in popular and rejected preschoolers. Br. J. Dev. Psychol. 20, 205-224. doi: 10.1348/026151002166415

Peterson, C. C., and Slaughter, V. P. (2006). Telling the story of theory of mind: deaf and hearing children's narratives and mental state understanding. Br. J. Dev. Psychol. 24, 151-179. doi: 10.1348/026151005X60022

Petrocchi, S., Filipponi, C., Antonietti, C., Levante, A., and Lecciso, F. (2021). Theory of mind as a mediator between emotional trust beliefs and interpersonal communication competence in a group of young adults. Psychol. Rep. 124, 555-576. doi: 10.1177/0033294120913489

Premack, D., and Woodruff, G. (1978). Premack and Woodruff: chimpanzee theory of mind. Behav. Brain Sci. 4, 515-526.

Ranta, K., La, A. M., and Riittakerttu, G. (2016). Social phobia and educational and interpersonal impairments in adolescence: A prospective study. Child Psychiatry Hum. Dev. 47, 665-677. doi: 10.1007/s10578-015-0600-9

Razza, R. A., and Blair, C. (2009). Associations among false-belief understanding, executive function, and social competence: A longitudinal analysis. J. Appl. Dev. Psychol. 30, 332-343. doi: 10.1016/j.appdev.2008.12.020

Rohner, R. P., and Khaleque, A. (2010). Testing central postulates of parental acceptance-rejection theory (PAR theory): A meta-analysis of cross cultural studies. J. Fam. Theory Rev. 2, 73-87. doi: 10.1111/j.1756-2589.2010.00040.x

Rotenberg, K. J. (ed.) (2010). "The conceptualization of interpersonal trust: a basis, domain, and target framework," in Interpersonal Trust During Childhood and Adolescence. (Cambridge University Press), 2-27.

Schofield, T. J., Conger, R. D., Donnellan, M. B., Jochem, R., Widaman, K. F., and Conger, K. J. (2012). Parent personality and positive parenting as predictors of positive adolescent personality development over time. MerrillPalmer Q. 58, 255-283. doi: 10.1353/mpq.2012.0008

Walker, S. U. E. (2005). Gender differences in the relationship between young children's peer-related social competence and individual differences in theory of mind. J. Gen. Psychol. 166, 297-312. doi: 10.3200/GNTP.166.3.297-312

Weber, L. R., and Carter, A. (1998). On constructing trust: temporality, selfdisclosure, and perspective-taking. J. Sociol. Soc. Pol. 18, 7-26.

Wei, Y. (2005). The confidence validity evaluation applied in the interpersonal ability questionnaire among college students. Chin. J. Sch. Heal. 26, 10461048. doi: 10.3969/j.issn.1000-9817.2005.12.036

Wellman, H. M. (2017). "Learning a theory of mind," in Theory of Mind Development in Context. eds. V. Slaughter and M. DeRosnay (London, UK: Routledge), 1-22.

Wu, L-h. (2014). The development of adult psychology theory and its relationship with interpersonal trust. Adult. Edu. 34, 28-30. doi: 10.3969/j. issn.1001-8794.2014.05.007

Xia, L. X., Ding, C., Hollon, S. D., and Yi, Y. (2015). Interpersonal self-support, venting coping and post-traumatic stress disorder symptoms among adolescent earthquake survivors. Curr. Psychol. 34, 14-25. doi: 10.1007/s12144-014-9237-2

Xiong, Q. (2015). Current situation and prospects of interpersonal relationship research for the deaf students in China. Mod. Spec. Edu. 20, 11-15.

Xiuqin, H., Huimin, Z., Mengchen, L., Jinan, W., Ying, Z., and Ran, T. (2010). Mental health, personality, and parental rearing styles of adolescents with internet addiction disorder. Cyberpsychol. Behav. Soc. Netw. 13, 401-406. doi: $10.1089 /$ cyber.2009.0222

$\mathrm{Xu}$, F. Z. (2018). The relationship between parental rearing and feeling of alienation among the deaf adolescents: moderated mediating effect. J. Sdnu. 63, 83-91.

$\mathrm{Xu}$, Q., and Ge, M. G. (2010). The influence of the development degree of college students' "theory of mind" on interpersonal relationship. Psychol. Res. 3, 53-57.

Zhang, L., and Eggum-Wilkens, N. D. (2018). Unsociability in Chinese adolescents: cross-informant agreement and relations with social and school adjustment. Soc. Dev. 27, 555-570. doi: 10.1111/sode.12284

Conflict of Interest: The authors declare that the research was conducted in the absence of any commercial or financial relationships that could be construed as a potential conflict of interest.

Publisher's Note: All claims expressed in this article are solely those of the authors and do not necessarily represent those of their affiliated organizations, or those of the publisher, the editors and the reviewers. Any product that may be evaluated in this article, or claim that may be made by its manufacturer, is not guaranteed or endorsed by the publisher.

Copyright (c) $2021 \mathrm{Wu}$, Liu, Zhang and Zhong. This is an open-access article distributed under the terms of the Creative Commons Attribution License (CC BY). The use, distribution or reproduction in other forums is permitted, provided the original author(s) and the copyright owner(s) are credited and that the original publication in this journal is cited, in accordance with accepted academic practice. No use, distribution or reproduction is permitted which does not comply with these terms. 